\title{
Обобщенная статистико-термодинамическая
} модель гетероассоциации ароматических
молекул в водном растворе для анализа
данных ЯМР-спектроскопии

\author{
Севастопольский посударственный технический университет Министерства просвещения Украины \\ Студгородок, Севастополь, 99053, Украина \\ 1 Беркбех колледж Лондонскопо университета \\ Гордон Хауз, 29, Лондон WС1Н ОРP, Великобритания
}

Д. А. Веселков ${ }^{1}$, М. П. Евстигнеев, Д. Б. Дэвис ${ }^{1}$, В. В. Кодинцев, А. Н. Веселков

\begin{abstract}
На основе базовой модели гетероассоциаци ароматииеских молекул в водном растворе разработана обобиенная статистико-термодинамическая модель для интерпретации данных ЯМР-спектроскопии. В предложенной модели, в отличие от базовой, учтены краевые эффекты, т. $e$. зависимость химического сдвига протонов от полохения молекулы в агрегате - внутри, на краях или в гетеростыке. Обе модели апробированы на примере гетероассочиации акридинового красителя профлавина (РF) и фенантридинового красителя бромистого этидия (EB) в водном растворе. Параметры ассоциации молекул рассиитывали по данньм 'Н-ЯМР спектроскопии (500 МГц). Исследованы әкспериментальные концентрационные и температурные зависимости протонньх химических сдвигов взаимодействуючих ароматических молекул. Установлено, что рассиитанные параметры по базовой и обобщенной моделям отличаются приблизительно на $30 \%$ и суцественно зависят от велииины равновесной константы гетероассоциачии КС - при высоких значениях КС погрешность базовой модели возрастает. Анализ структурных и термодинамических характеристик комплексообразования РF и ЕВ позволяет сделать вывод о преимуцественном вкладе дисперсионньх взаимодействий в стабилизаиию гетерокомплекса PF-EB.
\end{abstract}

Введение. Исследования гетероассоциации ароматических молекул и их конкурентного связывания с рецепторами - ДНК или белками - важны по двум причинам. Во-первых, они позволяют получить информацию о природе физико-химических взаимодействий, влияющих на сродство ароматических молекул в растворе, обусловленных как структурными особенностями хромофора, так и боковых цепей ароматического лиганда. Во-вторых, с медико-биологической точки зрекия гетерокомплексы ароматических молекул и их конкурентное связывание с рецепторами могут оказывать влияние на эффективность действия антибиотиков. $\mathrm{K}$ этой проблеме можно отнести, например, взаимодействие антибиотиков $\mathrm{c}$ ароматическими молеку-

(C) Д. А. ВЕСЕЛКОВ, М. П. ЕВСТИГНЕЕВ, Д. Б. ДЭВИС, в. В. КОДИнLЕВ, А. Н. ВЕСЕЛКОВ, 2001 лами из пищевых источников (полифенолы, метилксантины типа кофеина и т. п.), а также вопросы применения антибиотиков при их комбинации [1, 2]. Следовательно, исследования гетероассоциации ароматических молекул имеет отношение к эффективности действия антибиотиков и диете при химиотерапии [3, 4]. Подобные исследования также необходимы для количественного анализа эффекта конкурентного связывания различных ароматических молекул с рецепторами, а также для установления молекулярного механизма действия ароматических соединений в качестве регуляторов фармакологической активности антибиотиков и как протекторов ДНК от связывания с ароматическими молекулами мутагенов [5-.8].

Статистико-термодинамическая модель гетероассоциации, в которой молекулы образуют бесконечномерные агрегаты как для самоассоциации 
[9], так и для гетероассоциации, была недавно разработана в нашей лаборатории [10] для анализа данных ЯМР ароматических молекул в смешанных растворах. Эффективность этой модели была продемонстрирована на примере гетероассоциации кофеина, одной из наиболее часто встречающихся «пищевых» ароматических молекул, с различными антибиотихами и мутагенами [8]. Необходимо, однако, отметить, что аналитические выражения, приведенные в модели [10], применимы только в том случае, когда константы самоассоциации взаимодействующих молекул существенно различаются (примерно на порядок). Вместе с тем исследование самоассоциации ароматических лигандов в водном растворе свидетельствует [9] о широком диапазоне величин равновесных констант самоассоциации молекул, отличающихся структурой хромофора и боковых цепей. В связи с этим для анализа гетероассоциации ароматических молекул с различными медико-биологическими свойствами (антибиотикантибиотик, антибиотик-мутаген), статистикотермодинамическая модель [10] должна быть обобщена на тот случай, когда нет ограничений на равновесные константы самоассоциации взапмодействующих молекул.

В настоящей работе рассмотрены базовая и обобденная модели гетероассоциации ароматических молекул, в которой дополнительно учтено влияние краевых эффектов при образовании различных молекулярных ассоциатов в растворе. Сравнительный анализ базовой и обобщенной моделей проведен на примере процесса гетероассоциации двух ароматических красителей - акридинового красителя профлавина (PF) и фенантридинового красителя бромистого этидия (ЕВ), которые обладают мутагенными свойствами (рис. 1).

ЯМР-спектроскопия (500 МГц) использована для определения термодинамических и структурных параметров комплексообразования молекул, рассчитываемых по экспериментальным зависимостях протонных химических сдвитов молекул как функции концентрации и температуры $[9,10]$.

Методика. Бромистый этидий и профлавин («Sigma», США) использовали без дополнительной очистки, растворяли в $\mathrm{D}_{2} \mathrm{O}$ с изотопной чистотой 99,95\% D («Sigma») и лиофилизировали. Растворы готовили, добавляя взвешенное количество образца в дейтерированном $0,1 \mathrm{M}$ фосфатном буфере (pD $7,1)$, содержащем $10^{-4}$ моль/л ЭДТА. Концентрацию ароматических молекул в водном растворе определяли спектрофотометрически - для ЕВ коэффициент экстинкции $\varepsilon=5860 \mathrm{M}^{-1} \mathrm{cM}^{-1} \quad(\lambda=$ $=480 \mathrm{нм})[11]$, для $\mathrm{PF}-\varepsilon=41000 \mathrm{M}^{1} \mathrm{cM}^{-1} \quad(\lambda=$ $=444 \mathrm{HM})[12]$.<smiles></smiles><smiles>CC[n+]1c(-c2ccccc2)c2cc(N)ccc2c2ccc(N)cc21</smiles>

Рис. 1. Структурные формулы молекул профлавина (a) и бромистого этидия (6) с ухазанием необменивающихся протонов

1M- и 2M- ${ }^{1}$ H-ЯMP спектры измерены на спектрометре «Bruker DRX» (Великобритания) с резонансной частотой 500 МГц. Остаточный сигнал HOD насыщался в период детектирования. Концентрационные измерения протонных химических сдвигов молекул выполнены при температурах 298 и $308 \mathrm{~K}$; температурные зависимости химических сдвигов протонов ароматических лигандов измерены в диапазоне температур от 278 до $358 \mathrm{~K}$. В процессе измерений концентрационных зависимостей концентрацию ЕВ поддерживали постоянной $\left(y_{0}=0,8 \mathrm{MM}\right)$ при изменении процентного содержания PF в растворе. Это связано с тем, что равновесная хонстанта самоассоциации молекул РF более чем в два раза превышает таковую для ЕВ $\left(K_{P F}=700 \mathrm{M}^{-1}, K_{E B}=310 \mathrm{M}^{-1}, T=298 \mathrm{~K}[9]\right)$ и, следовательно, изменение концентрации акридинового красителя оказывает большее влияние на равновесное распределение агрегатов, чем вариация содержания бромистого этидия в растворе. Все ЯМР 
измерения выполнены в условиях быстрого обмена взаимодействующих молекул во временном масштабе ЯМР. Химический сдвиг определяли относительно ДСС (2,2-диметил-2-силапентан-5-сульфокислота), в качестве внутреннего стандарта использовали бромистый тетраметиламмоний (ТМА). Отнесение сигналов в спектрах ${ }^{1} \mathrm{H}$-Я्रМР, отождествление химических и пространственных связей проводили соответственно с помощью двухмерных гомоядерных TOCSY и ROESY экспериментов. Mетодика приготовления образцов и проведения экспериментов описана в $[10,13]$.

Результаты и обсуждение. Базовал модель гетероассоциации ароматических молекул. В модели молекулярной гетероассоциации [10] двух веществ А и Р предполагается существование динамического равновесия в растворе, включающего реакции бескокечномерной самоассоциации и гетероассоциации различных типов хомплексов:

$$
\begin{aligned}
& A_{1}+A_{i} \stackrel{K_{A}}{\leftrightarrow} A_{i+1} \quad(a) ; \quad P_{1}+P_{j} \stackrel{K_{p}}{\leftrightarrow} P_{j+1} \\
& A_{i}+P_{j} \stackrel{K_{C}}{\leftrightarrow} A_{i} P_{j} \quad \text { (в); } \quad P_{j} A_{i}+P_{i} \stackrel{K_{C}}{\leftrightarrow} P_{j} A_{i} P_{\mathrm{l}} \\
& A_{k}+P_{j} A_{i} \stackrel{K_{C}}{\leftrightarrow} A_{i} P_{j} A_{k} \quad \text { (d). }
\end{aligned}
$$

где $A_{1}, P_{1}$ - концентрации мономеров веществ $A$ и P соответственно; $A_{i}, A_{k}, P_{j}, P_{l}$ - агрегаты, содержащие $i, k$ мономеров $A$ и $j, l$ мономеров $P$. Равновесные константы реакций самоассоциации $K_{A}$ и $K_{p}$ и гетероассоциации $K_{C}$ полагаются независимыми от числа молекул в агрегатах и комплексах. В модели, использованной ранее [10], реакцию $1, \partial$, не учитывали ввиду большого различия равновесных констант самоассоциации $\left(K_{A}>K_{P}\right)$ для исследованных ароматических молекул. В общем случае, когда константы самоассоциации молекул близки по величине, учет реакции $1, \partial$, необходим. К этому следует добавить, что перед суммой бесконечных рядов для симметричных гетерокомплексов, например, $P_{j} A_{i} P_{i}$ или $A_{i} P_{j} A_{k}$ должен стоять коэффициент $1 / 2$, чтобы одинаковые типы комплексов не учитывать в расчетах дважды. Таким образом, используя подход, аналогичный описанному ранее $[10]$, закон сохранения массы для случая бесконечномерной ассоциации ароматических молекул в реакциях (1) может быть записан в следующей форме:

$$
a_{0}=\frac{a_{1}}{\left(1-K_{A} a_{1}\right)^{2}}\left[1+K_{C} \frac{p_{1}}{1-K_{P} p_{1}}+\right.
$$

$$
\begin{gathered}
\left.+\frac{K_{C}^{2}}{2} \frac{p_{1}^{2}}{\left(1-K_{p} p_{1}\right)^{2}}+K_{C}^{2} \frac{a_{1} p_{1}}{\left(1-K_{A} a_{1}\right)\left(\left(1-K_{P} p_{1}\right)\right.}\right] \\
p_{0}=\frac{p_{1}}{\left(1-K_{p} p_{1}\right)^{2}}\left[1+K_{C} \frac{a_{1}}{1-K_{A} a_{1}}+\right. \\
\left.+\frac{K_{C}^{2}}{2} \frac{a_{1}^{2}}{\left(1-K_{A} a_{1}\right)^{2}}+K_{C}^{2} \frac{a_{1} p_{1}}{\left(1-K_{A} a_{1}\right)\left(\left(1-K_{P} p_{1}\right)\right.}\right]
\end{gathered}
$$

где $a_{0}, p_{0}$ и $a_{1}, p_{1}-$ исходные и мономерные молярные концентрации.

Зависимость наблюдаемых протонных химических сдвигов в такой модели может быть записана в симметричной форме по отношению к индексам «A» и «P». Для вещества $A$ :

$$
\begin{aligned}
\delta_{A}= & \frac{a_{1}}{a_{0}}\left[\delta_{m A}\left(2\left(1+K_{A} a_{1}\right)-\frac{1}{\left(1-K_{A} a_{1}\right)^{2}}\right)+\right. \\
& +2 \delta_{d A}\left(\frac{1}{\left(1-K_{A} a_{1}\right)^{2}}-1-K_{A} a_{1}\right)+ \\
& +\delta_{C A} \frac{K_{C} p_{1}}{\left(1-K_{A} a_{1}\right)^{2}\left(1-K_{P} p_{1}\right)} \times \\
& \left.\times\left(1+\frac{K_{C} p_{1}}{2\left(1-K_{P} p_{1}\right)}+\frac{K_{C} a_{1}}{1-K_{A} a_{1}}\right)\right]
\end{aligned}
$$

и соответственно для $P$ :

$$
\begin{aligned}
\delta_{P}= & \frac{p_{1}}{p_{0}}\left[\delta_{m^{p}}\left(2\left(1+K_{p} p_{1}\right)-\frac{1}{\left(1-K_{P} p_{1}\right)^{2}}\right)+\right. \\
& +2 \delta_{d P}\left(\frac{1}{\left(1-K_{P} p_{1}\right)^{2}}-1-K_{p} p_{1}\right)+ \\
& +\delta_{C P} \frac{K_{C} a_{1}}{\left(1-K_{p} p_{1}\right)^{2}\left(1-K_{A} a_{1}\right)} \times \\
& \left.\times\left(1+\frac{K_{C} a_{1}}{2\left(1-K_{A} a_{1}\right)}+\frac{K_{C} p_{1}}{1-K_{p} p_{1}}\right)\right] .
\end{aligned}
$$

Величины $\delta_{m A}, \delta_{d A}, \delta_{m p}, \delta_{d P}$ и равновесные константы $K_{A}$ и $K_{P}$ определяются из независимых экспериментов в идентичных экспериментальных условиях. Отсюда следует, что наблюдаемые концентрационные зависимости протонных химических сдвигов ароматических молекул веществ $A$ и $P$ в смешанных растворах являются функцией двух неизвестных параметров $\delta_{c}$ и $K_{C}$, которые могут быть найдены с использованием вычислительной процедуры, описанной ранее [10].

Рассмотренная модель гетероассоциации снимает ограничения на равновесные константы самоассоциации взаимодействующих молекул и позволяет получить аналитические выражения для про- 
тонного химического сдвига в сравнительно простом функциональном виде. Это достигается введением допущений в модель динамического равновесия молекул, исключающих из рассмотрения краевые эффекты, т. е. зависимости химического сдвига протонов от положения молекулы в агрегате внутри, на краях или в гетеростыке [10].

Обобщенная модель гетероассоциации ароматических молекул. Так же, как и в базовой модели, рассматривается схема реакций (1) в растворе. Концентрации образующихся комплексов, вычисленных по закону действующих масс, можно представить в виде:

$$
\begin{gathered}
x_{i+1}=K_{X} x_{i} x_{1}=K_{X}^{i} x_{1}^{i+1} \\
y_{j+1}=K_{Y} y_{j} y_{1}=K_{Y}^{j} y_{1}^{j+1} \\
z_{i j}=K_{C} x_{i} y_{j}=K_{C} K_{X}^{i-1} K_{Y}^{j-1} x_{1}^{i} y_{1}^{j} \\
\omega_{j i l}=K_{C}^{2} y_{j} x_{i} y_{l}=K_{C}^{2} K_{Y}^{j-1} K_{X}^{i-1} K_{Y}^{d-1} y_{1}^{j} x_{1}^{i} y_{1}^{j} \\
\alpha_{i j k}=K_{C}^{2} x_{i} y_{j} x_{k}=K_{C}^{2} K_{X}^{i-1} K_{Y}^{j-1} K_{X}^{k-1} x_{1}^{i} y_{1}^{j} x_{1}^{k} .
\end{gathered}
$$

Уравнения (2), (3), выражающие закон сохранения массы, предполагают суммирование компонент по всем индексам, например, для комплекса $Y_{j} X_{i} Y_{i}$

$$
\sum_{j=1}^{\infty} \sum_{i=1}^{\infty} \sum_{i=1}^{\infty} \omega_{j i l}
$$

Индексы $j, i, l$ независимы друг от друга, поэтому среди слагаемых в (7) $c j \neq 1$ всегда найдется пара симметричных друг другу с равными концентрациями $\omega_{j i l}=\omega_{l i j}$, соответствующих тождественным в растворе комплексам $Y_{j} X_{i} Y_{l}$ и $Y_{l} X_{i} Y_{j}$ Следовательно, сумма (7) должна быть скорректирована к виду:

$$
\frac{1}{2}\left(\sum_{i=1}^{\infty} \sum_{i=1}^{\infty} \sum_{i=1}^{\infty} \omega_{j i l}+\sum_{j=1}^{\infty} \sum_{i=1}^{\infty} \omega_{j i j}\right) .
$$

Тогда закон сохранения массы для рассматриваемой системы можно записать следующим образом:

$$
\begin{gathered}
x_{0}=\sum_{i} i x_{i}+\sum_{i} \sum_{i} i z_{i j}+\frac{1}{2}\left(\sum_{i} \sum_{j} \sum_{i} i \omega_{j i l}+\sum_{j} \sum_{i} i \omega_{j i j}\right)+ \\
+\frac{1}{2}\left(\sum_{k} \sum_{j} \sum_{i}(i+m) a_{i j m}+\sum_{j} \sum_{i} 2 i a_{i j i}\right) \\
y_{0}=\sum_{j} j y_{j}+\sum_{i} \sum_{j} j z_{i j}+ \\
+\frac{1}{2}\left(\sum_{i} \sum_{l} \sum_{j}(j+l) \omega_{j i}+\sum_{i} \sum_{j} 2 j \omega_{j i j}\right)+
\end{gathered}
$$

$$
+\frac{1}{2}\left(\sum_{k} \sum_{i} \sum_{j} j a_{i j m}+\sum_{i} \sum_{j} j a_{i j i}\right),
$$

где $x_{0}, y_{0}$ - исходные молярные концентрации веществ $X$ и $Y$. Произведя суммирование бесконечных рядов с учетом (6), можно представить закон сохранения массы в матричном виде:

$$
x_{0}=\left[K_{0}\right] \cdot[C] \text {, }
$$

где

$$
\left[K_{0}\right]=\left(\frac{1}{1-K_{x} x_{1}} \frac{1}{1-K_{X} x_{1}} \frac{1}{1-K_{x} x_{1}} \frac{K_{X}^{2} x_{1}^{2}+K_{X} x_{1}+2}{1-K_{x}^{2} x_{1}^{2}}\right)
$$

- матрица коэффициентов;

$$
[C]^{T}=\left(\left[X_{i}\right]\left[X_{i} Y_{j}\right]\left[Y_{j} X_{i} Y_{l}\right]\left[X_{i} Y_{j} X_{k}\right]\right)
$$

- матрица концентраций типов комплексов;

$$
\begin{gathered}
{\left[X_{i}\right]=\frac{x_{1}}{1-K_{X} x_{1}} ;\left[Y_{j}\right]=\frac{y_{1}}{1-K_{Y} y_{1}} ;} \\
\left.\left[X_{i} Y_{j}\right]=K_{C}\left[X_{i}\right] Y_{j}\right] ; \\
{\left[Y_{i} X_{i} Y_{l}\right]=\frac{K_{C}^{2}\left[X_{i}\right]\left[Y_{j}\right]^{2}}{1+K_{Y} y_{1}} ;\left[X_{i} Y_{j} X_{k}\right]=\frac{K_{C}^{2}\left[X_{i}\right]^{2}\left[Y_{j}\right]}{1+K_{X} x_{i}}}
\end{gathered}
$$

Система уравнений (9) симметрична относительно $X$ и $Y$ и, следовательно, закон сохранения массы для вещества $Y$ получается из (10) простой заменой индексов $\boldsymbol{x}$ на $\boldsymbol{y}$ и наоборот.

Анализ зависимости протонного химического сдвига молекул от концентрации и температуры раствора предполагает быстрый обмен между различными ассоциатами в масштабе времени ЯМР и учет вклада только ближайщих соседних молекул в эхранирование исследуемых протонов. Если агрегат образован в результате самоассоциации молекул одного сорта, то вклад соседних молекул в экранирование ядер полагается одинаковым [10]:

$$
\delta_{i}-\delta_{m}=2\left(\delta_{d}-\delta_{m}\right),
$$

где $\delta_{i}, \delta_{d}, \delta_{m}-$ соответственно химические сдвиги протона внутри самоассоциата, на краю (или в димере) и в мономере.

Для того чтобы учесть влияние положения молекулы в агрегате на химический сдвиг протонов, необходимо рассмотреть все возможные виды ее ближайтего окружения, т. е. какого типа контакты (стыки) образует молекула с соседями. Пусть $\Delta \delta_{s}$ и $\Delta \delta_{c}$ - добавки в химический сдвиг соответственно от соседней молекулы одного и того же вещества (гомостык) и вецества другого сорта (гетеростык). Тогда химический сдвиг может принимать следующие значения: a) $\delta_{m}-$ в мономере; 
б) $\delta_{d}=\delta_{m}+\Delta \delta_{s}-$ на краю агрегата с гомостыком; в) $\delta_{c}=\delta_{m}+\Delta \delta_{c}-$ на краю агрегата с гетеростыком; г) $\delta_{i}=\delta_{m}+2 \Delta \delta_{s}-$ внутри агрегата с двумя гомостыхами; д) $\delta_{c c}=\delta_{m}+2 \Delta \delta_{c}-$ внутри агрегата с двумя гетеростыками; е) $\delta_{i C}=\delta_{m}+\Delta \delta_{s}+\Delta \delta_{C}=\delta_{d}+$ $+\Delta \delta_{c}-$ внутри агрегата с гетеро- и гомостыком соответственно. Принимая во внимание, что вклад в экранирование ядер дает лишь ближайшее окружение молекулы, можно привести все вероятные виды комплексов, различающиеся по химическому сдвигу (табл. 1). Тогда аддитивная модель для наблюдаемого химического сдвига протона может быть представлена в виде суммы вкладов от различных видов комплексов:

$$
\delta_{X}=\sum_{i=1}^{9} D_{i} .
$$

Группируя значения $D_{i}$ по типам комплексов $\left(D_{12}\left(X_{i}\right)=D_{1}+D_{2}, \quad D_{34}\left(X_{i} Y_{j}\right)=D_{3}+D_{4}, \quad D_{56(} Y_{j} X_{i} Y_{l}\right)=$ $=D_{5}+D_{6}, D_{789}\left(X_{i} Y_{f} X_{k}\right)=D_{7}+D_{8}+D_{9}$ (табл. 1)), можно записать выражение для химического сдвига вещества $X$ в обобщенном матричном виде:

$$
\delta_{x}=\left[\delta_{X}\right] \cdot\left[K_{1}\right] \cdot\left[K_{2}\right]
$$

где

$$
\begin{aligned}
& {\left[\delta_{X}\right]=\left[\delta_{m X} \delta_{d X} \delta_{C X}\right] \text {, }} \\
& \begin{array}{llll}
D_{12} & D_{34} & D_{56} & D_{789}
\end{array}
\end{aligned}
$$

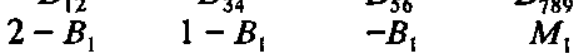

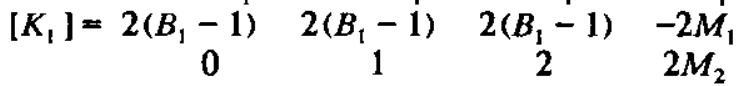

$$
\begin{aligned}
& \begin{array}{c}
B_{0} B_{1} \\
B_{0} B_{C} B_{1} B_{2} \\
\left.K_{2}\right]=B_{C}{ }^{2} B_{0} B_{l} B_{2}{ }^{2} B_{3} \\
B_{C}{ }^{2} B_{0} B_{2}
\end{array} \\
& B_{0}=\frac{x_{1}}{x_{0}}, B_{C}=K_{C}, B_{1}=\frac{1}{1-K_{X} x_{1}}, \\
& B_{2}=\frac{y_{1}}{1-K_{\gamma} y_{1}}, B_{3}=\frac{1}{1+K_{Y} y_{1}}, \\
& M_{1}=x_{1}\left[\frac{2 B_{1}^{3}}{2 B_{1}-1}-B_{1}^{3}+\frac{\left(B_{1}-1\right)^{3}}{B_{1}}-\frac{B_{1}^{4}}{\left(2 B_{1}-1\right)^{2}}\right] \text {, } \\
& M_{2}=x_{1} \frac{B_{1}^{3}}{2 B_{1}-1} \text {. }
\end{aligned}
$$

Очевидно, что для протонов вещества $Y$ значение химического сдвига получается из (12) простой заменой индексов $x$ на $y$ и наоборот.

Удобство записи (12) состоит в следующем: а) вынисление произведений матриц в последовательности $\delta_{X}=\left[\delta_{X}\right] \cdot\left(\left[\mathbf{K}_{1}\right] \cdot\left[\mathbf{K}_{2}\right]\right)$ дает три слагаемых, отражающих вклад в химический сдвиг параметров $\delta_{m X}, \delta_{d X}, \delta_{C X}$

$$
\begin{gathered}
\delta_{X}=\delta_{m X}\left[B_{0} B_{1}\left(2-B_{1}\right)+B_{0} B_{c} B_{1} B_{2}\left(1-B_{1}\right)-\right. \\
\left.-B_{C}^{2} B_{0} B_{1}^{2} B_{2}^{2} B_{3}+B_{C}^{2} B_{0} B_{2} M_{1}\right]+ \\
+\delta_{d X}\left[2 B_{0} B_{1}\left(B_{1}-1\right)+2 B_{0} B_{c} B_{1} B_{2}\left(B_{1}-1\right)+\right. \\
\left.+2 B_{C}^{2} B_{0} B_{1} B_{2}^{2} B_{3}\left(B_{1}-1\right)-2 B_{c}^{2} B_{0} B_{2} M_{1}\right]+ \\
+\delta_{C X}\left[B_{0} B_{C} B_{1} B_{2}+2 B_{C}^{2} B_{0} B_{1} B_{2}^{2} B_{3}+2 B_{C}^{2} B_{0} B_{2} M_{2\}} ;\right.
\end{gathered}
$$

б) вычисление произведений в последовательности $\delta_{X}=\left(\left[\delta_{X}\right] \cdot\left(\left[\mathrm{K}_{1}\right]\right) \cdot\left[\mathrm{K}_{2}\right.\right.$ цает четыре составляющих, отражающих влияние на химический сдвиг конкретных типов комплексов $-D_{12}\left(X_{i}\right), D_{34}\left(X_{i} Y_{j}\right)$, $D_{56}\left(Y_{j} X_{i} Y_{l}\right), D_{789}\left(X_{i} Y_{j} X_{k}\right)$ :

$$
\begin{gathered}
\delta_{X}=B_{0} B_{1}\left[\delta_{m X}\left(2-B_{1}\right)+2 \delta_{d X}\left(B_{1}-1\right)\right]+ \\
\left.+B_{0} B_{C} B_{1} B_{2}\left[\delta_{m X}\left(1-B_{1}\right)+2 \delta_{d X}\left(B_{1}-1\right)\right]+\delta_{C X}\right]+ \\
+B_{C}^{2} B_{0} B_{1} B_{2}^{2} B_{3}\left[-\delta_{m X} B_{1}+2 \delta_{d X}\left(B_{1}-1\right)+2 \delta_{C X}\right]+ \\
+B_{C}^{2} B_{0} B_{2}\left[\delta_{m X} M_{1}-2 \delta_{d X} M_{1}+2 \delta_{C X} M_{2}\right]
\end{gathered}
$$

Вычислительная процедура нахождения параметров модели соответствует описанной ранее [10]. Входными данными являются экспериментальные концентращионные и температурные зависимости химических сдвигов ароматических протонов исследуемого вещества, а также результат расчета параметров самоассоциации обоих веществ: $\delta_{m x}$, $\delta_{d X}, K_{X} ; \delta_{m Y}, \delta_{d \gamma}, K_{Y} ;$ выходными $-\delta_{c X}, \delta_{c \gamma}, K_{c}$ и энтальпия $(\Delta H)$, энтропия $(\Delta S)$, определяемые исходя из концентрационных и температурных зависимостей соответственно.

Квадратичная функция невязки между экспериментальными и теоретическими значениями химического сдвига выступает целевой функцией процедуры минимизации по выходным параметрам, при этом на каждом цикле итерации решается система уравнений (10) для обоих веществ относительно $x_{1}$ и $y_{l}$, которые в дальнейшем используются B (12).

Сравнение результатов эксперимента по базовой и обобщенной моделям. Структурные и термодинамические параметры гетероассоциации РF и EB определяли путем анализа зависимостей химических сдвигов необменивающихся протонов обоих ароматических соединений в смешанном растворе от концентрации и температуры (рис. 2) по описанным выше базовой и обобщенной моделям, введя обозначения: $\mathrm{PF}-X, \mathrm{~EB}-Y$. В расчетах использованы параметры самоассоциации $\delta_{m X}, \delta_{d X}, K_{X}$ 


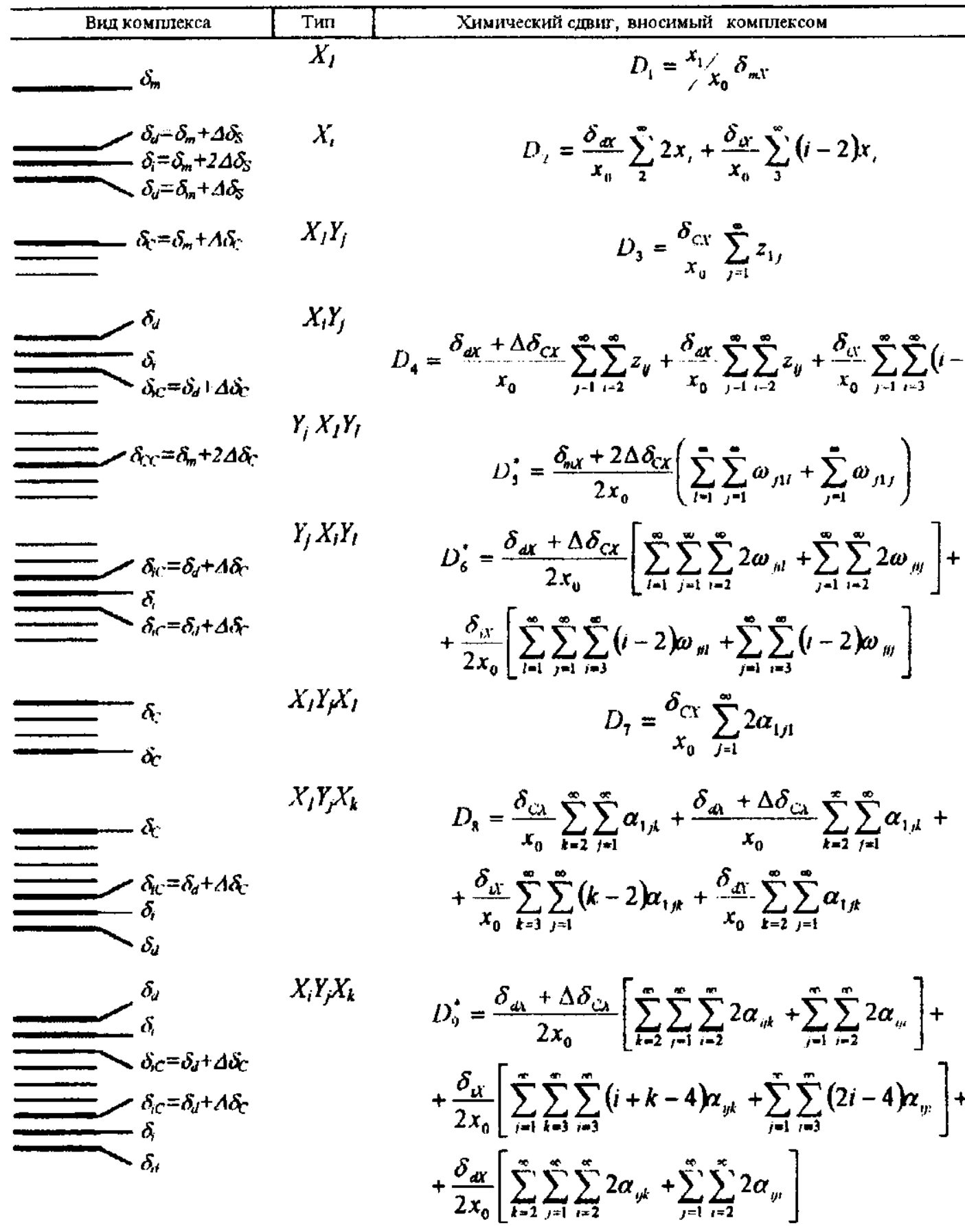



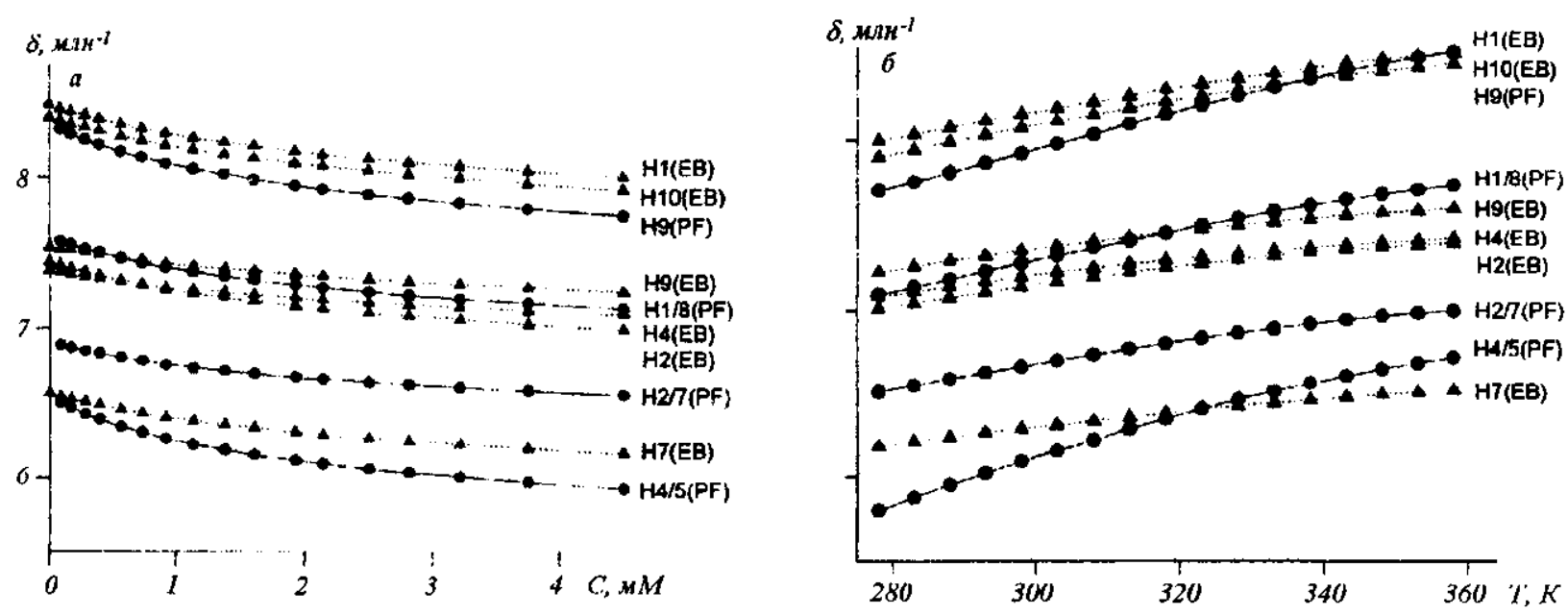

Рис. 2. Экспериментальные зависимости химических сдвигов протонов профлавина (РF) и бромистого этидия (ЕВ) в смешанном растворе: $a-$ or концентрации PF при $T-298 \mathrm{~K}, y_{0}=0,8$ мМ; 6 - от темлературы при $y_{0}=0,8$ мМ, $x_{0}=2,145$ мМ

таблина 2

Параметры гетероассоциации PF (X) и EB (Y) в 0,1 моль/л фосфатном буфере, рD 7, 1 , рассчитаннье по базовой нодели

\begin{tabular}{|c|c|c|c|c|c|}
\hline$T, \mathbf{k}$ & Протоны PF & $\delta_{C X}$ млн $^{-1}$ & KX, л/моль & $\Delta H$, кДж/моль & $\Delta S$, Дж/ (моль $K$ ) \\
\hline 298 & H9 & 7,64 & $690 \pm 60$ & $-(24,7 \pm 1,5)$ & $-(33,7 \pm 4,8)$ \\
\hline$K_{X}=700 \pm 70$ & $\mathrm{HI} / 8$ & 7,08 & - & - & 一 \\
\hline \multirow[t]{2}{*}{$K_{Y}-310 \pm 20$} & $\mathrm{H} 2 / 7$ & 6,56 & - & - & - \\
\hline & $\mathrm{H} 4 / 5$ & 6,03 & - & - & - \\
\hline 308 & $\mathrm{H} 9$ & 7,66 & $400 \pm 50$ & - & - \\
\hline$K_{X}=420 \pm 40$ & $\mathrm{H} 1 / 8$ & 7,11 & - & - & - \\
\hline \multirow[t]{2}{*}{$K_{Y}-230 \pm 20$} & $\mathrm{H} 2 / 7$ & 6,58 & - & - & - \\
\hline & $\mathrm{H} 4 / 5$ & 6,10 & - & - & - \\
\hline
\end{tabular}

и $\delta_{m Y}, \delta_{d \gamma}, K_{Y}$, определенные для РF и ЕВ ранее [9] в идентичных экспериментальных условиях. Результаты расчетов по базовой и обобщенной моделям представлены соответственно в табл. 2 и 3.

Сравнение результатов расчетов (табл. 2, 3) позволяет сделать вывод о том, что константы реакций и термодинамические параметры гетероассоциации молекул при анализе экспериментальных данных по двум моделям отличаются приблизительно на $30 \%$. Характерной особенностью полученных результатов является завышенное значение параметров гетероассоциации, вычисленных по базовой модели, в сравнении с параметрами, рассчитанными по обобщенной модели. Последнее связа- но с введением в обобщенную схему краевых эффектов, учитывающих положение молекулы в гетерокомплексе: в гомо- или гетеростыке. Это приводит к снижению мольной доли составляющей $\delta_{C}$ в выражении (13) по отношению к аналогичному выражению (3) базовой модели и, следовательно, к уменьшению общего вклада гетерокомплексов в динамическое равновесие в растворе. Отскда следует, что базовая модель будет отражать процесс гетероассоциации с тем меньшей погрешностью, чем меньше константа гетероассоциации в сравнении с константами самоассоциации молекул.

Для тестирования обобщенной модели на адекватность эксперименту использована также схема 
Tаблица 3

Параметры гетероассоциаци $P F(X)$ и $E B(Y)$ в 0,1 моль/л фосфатном буфере, pD 7,1, рассчитанные по обобщенной модели

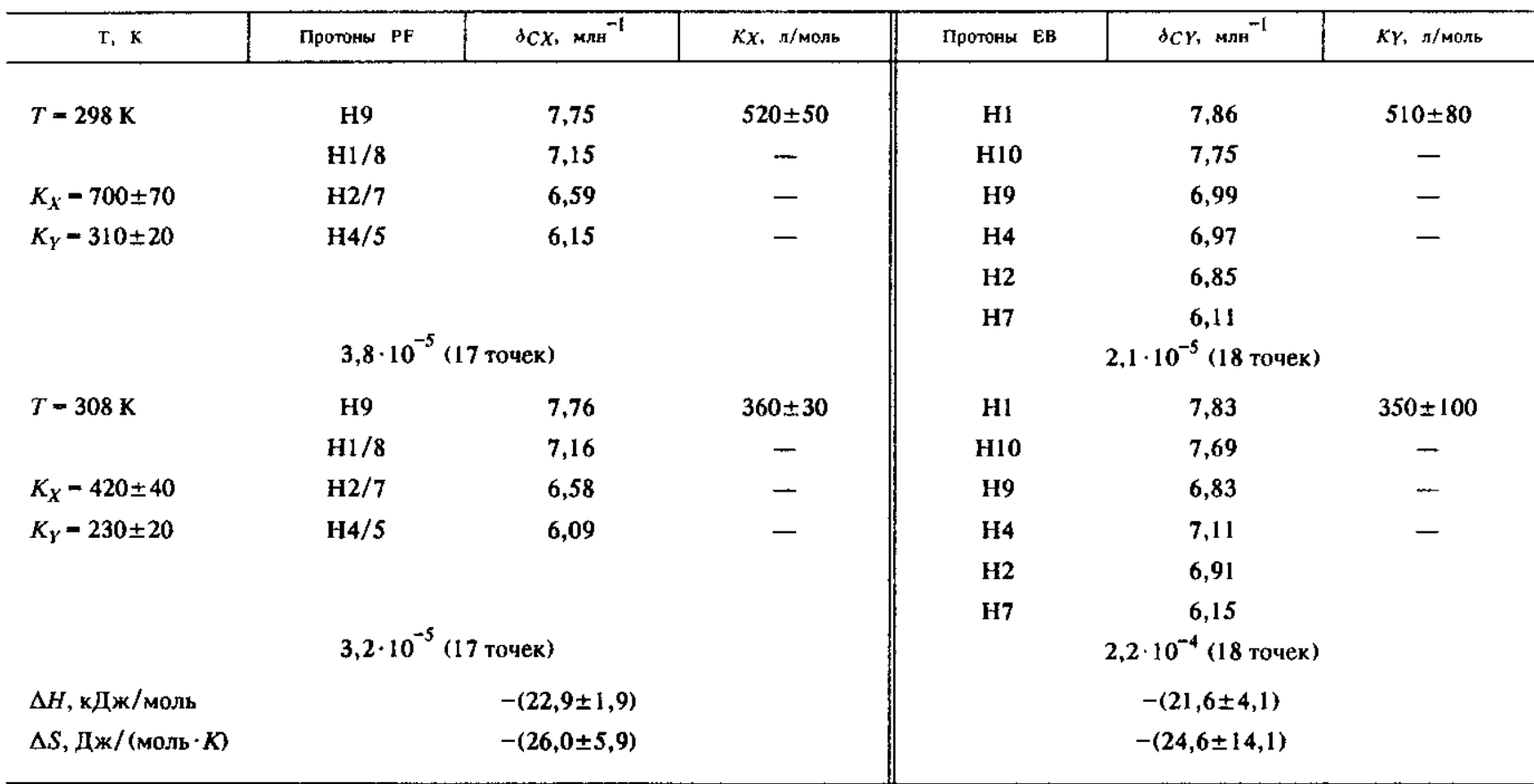

расчетов термодинамических параметров по концентрационным и температурным зависимостям химических сдвигов протонов ЕВ. При этом допускалось незначительное варьирование индуцированного химического сдвига $\delta_{d \gamma}$. Из данных табл. 3 следует, что равновесные константы, энтальпия и энтропия гетероассоциации, рассчитанные по данным эксперимента для двух различных красителей, совпадают в пределах погрешности расчета, что свидетельствует о самосогласованности использованкой модели.

Как видно из табл. 2 и 3, величины равновесных констант гетероассоциации РF и ЕВ не превышают константы самоассоциации профлавина, но заметно выше, чем соответствуюшая константа самоассоциации бромистого этидия. По-видимому, это связано с отсутствием боковых групп у хромофора молекулы PF, что создает более благоприятные стерические условия для образования димерного гетерокомплекса PF-EB, чем образование димера бромистого этидия, молекула которого содержит присоединенные к хромофору массивные фенольное кольцо и этильную группу.

Термодинамические параметры $\Delta H$ и $\Delta S$ реакции гетероассоциации PF и ЕВ определены на основе экспериментальных температурных зависимостей протонных химических сдвигов молекул по обеим моделям с использованием зависимости для константы гетероассоциации от температуры [9, 10]

$$
K_{c}(T)=\exp (\Delta S / R-\Delta H / R T)
$$

в предположении о том, что величины $\Delta H$ и $\Delta S$ практически не изменяются в исследованном диапазоне температур. Энтальпия образования гетероассоциата PF-EB имеет достаточно большое отрицательное значение, совпадающее в пределах погрешности с $\Delta H$ самоассоциации ЕВ в водном растворе $\left(\Delta H_{E B}=-(23,4 \pm 3,3)\right.$ кДж/моль [9]). Известно, что экзотермические реакции характерны для процессов агрегации, включающих в себя стэкинг-взаимодействие ароматических систем с делокализованными $\pi$-электронами $[14,15]$. Дисперсионные взаимодействия характеризуются как отрицательной энтальпией, так и отрицательной энтропией [16]. Следовательно, можно предположить, что дисперсионные взаимодействия дают основной вклад в стабилизацию PF-EB гетерокомплексов.

На рис. 3 приведены расчетные зависимости относительного содержания различных типов ассо- 

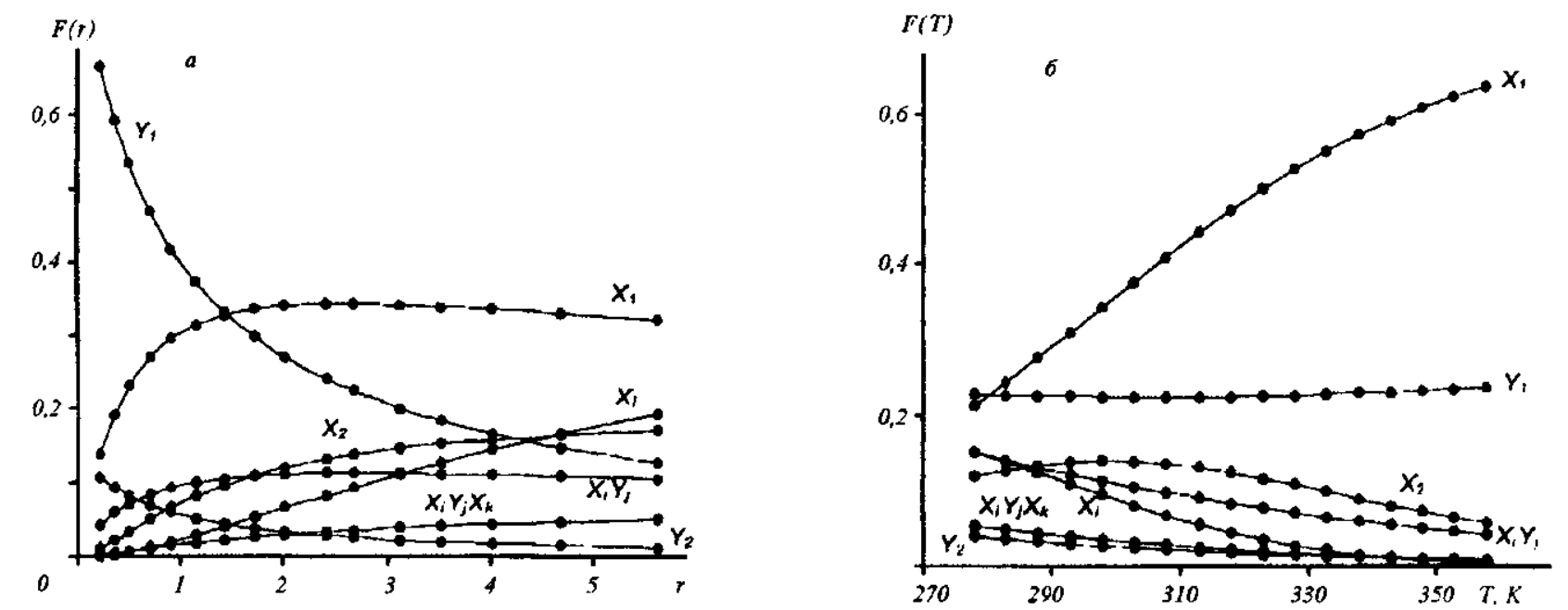

Рис. 3. Относительное содержание самоассоциатов и гетероассоциатов профлавина и бромистото этидия в зависимости от: $a-r-$ - $x_{0} / y_{0}$ - отношения концентраций PF и ЕB; 6 - температуры при $y_{0}=0,8$ мM, $x_{0}=2,145$ мM

циатов в смешанном растворе от отношения концентраций $\mathrm{PF}$ и $\mathrm{EB} r=x_{0} / y_{0}$ (рис. $3, a$ ) и температуры (рис. 3, б) (относительный вклад ассоциатов $Y_{j}$ и $Y_{j} X_{i} Y_{l}$ незначителен и на рисунке не отражен). Из рис. $3, a$, видно, что с ростом $r$ содержание мономерных и ассоциированных форм бромистого этидия уменьшается на фоне увеличения вклада ассоциированных форм профлавина: $X_{i}, X_{i} Y_{j} X_{k}$. Последнее приводит к появлению слабовыраженного максимума на кривых $X_{1}$ и $X_{i} Y_{j}$, причем содержание гетерокомплекса $X_{i} Y_{i}$ определяется конкурирующим взаимодействием $X_{i}$ и $Y_{j}$. Температурная зависимость относительного содержания типов комплексов (рис. 3, б) указывает на то, что при низких темдературах ароматические молекулы в основном находятся в ассоциированном состоянии. С повышением температуры содержание практически всех ассоциатов монотонно уменьшается, что сопровождается увеличением концентрации мономеров PF и EВ в смешанном растворе. Вместе с тем доля димеров профлавина $\left(X_{2}\right)$ имеет максимум в области температуры $300 \mathrm{~K}$. Такая зависимость для димерных агрегатов может быть объяснена тем, что n-мерные агрегаты PF и гетероассоциаты PF-EB разрушаются при более низких температурах в сравнении с димерными ассоциатами профлавина, что приводит первоначально к возрастанию содержания димеров PF с увеличением температуры и последующему его уменьшению вследствие их диссоциации в области высоких температур.

Структура $1: 1$ гетерокомплекса $P F-E B$ в водном растворе. Полученные по обобщенной модели значения химических сдвигов $\delta_{c}$ (табл. 3) для протонов PF и ЕВ были использованы для расчета наиболее вероятной структуры 1:1 комплекса профлавина с бромистым этидием в водном растворе. Взаимное расположение молекул в гетерокомплексе определяли путем установления соответствия значений индуцированных протонных химических сдвигов $\left(\Delta \delta=\delta_{m}-\delta_{c}\right)$ теоретическим кривым экранирования, рассчитанным квантовомеханически для РF и ЕВ [17]. Использование кривых экранирования как для PF, так и для ЕВ при построении структуры 1:1 гетероассоциата позволяет существенно повнсить точность ее определения в сравнении со структурами димерных комплексов при самоассоциации ароматических молекул [9].

На рис. 4 представлена найденная в результате расчетов наиболее вероятная пространственная структура 1:1 гетерокомплекса PF с EB в водном растворе. Пространственное изображение гетерокомплекса получено с помощью программы «Маthematica 2.2» (Wolfram Res. Inc.). Плоскости хромофоров молекул EB и PF в 1:1 гетероассоциате параллельны друг другу и расположены на расстоянии 0,34 нм; в таком комплексе имеет место довольно сильное перекрывание ароматических колец взаимодействующих молекул (стэкинг-взаимодействие), что предполагает существенную роль дисперсионных взаимодействий при образовании гетероассоциата.

Выводы. На основе базовой модели гетероассо- 


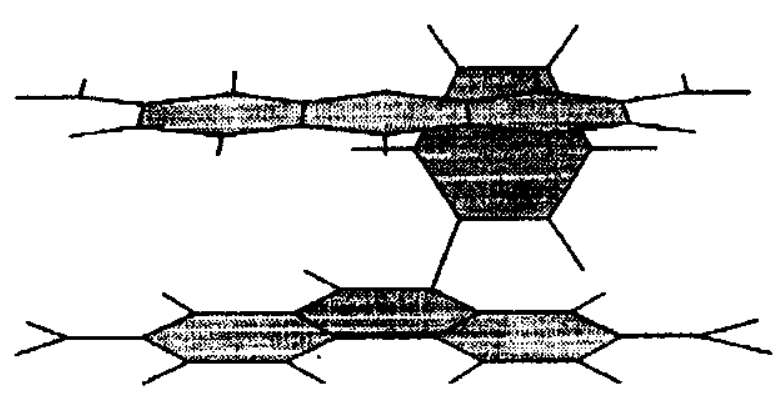

a

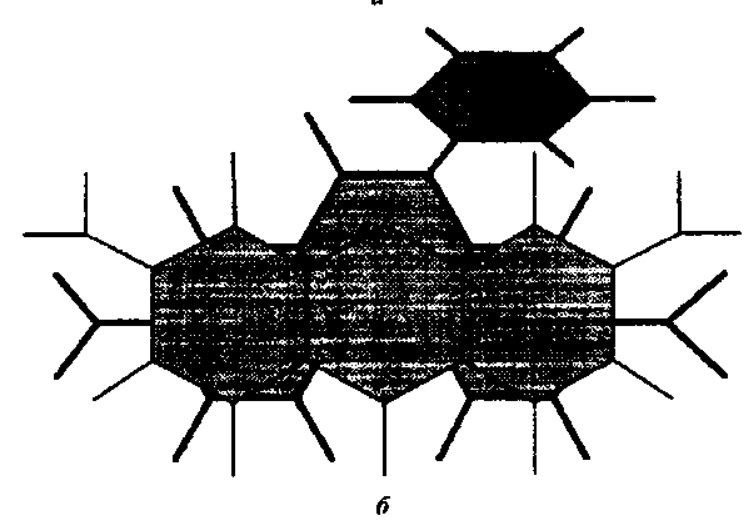

Рис. 4. Расчетная структура 1:1 гетерокомплекса профлавина и бромистопо этидия: $a$ - вид на комплекс сбоку; плоскости хромофоров молекул РF и ЕВ заштрихованы; 6 - вид сверху, показывающий взаимное расположение плоскостей хромофоров молекулы профлавина и бромистого этидня в гетерокомплексе

циации ароматических молекул в водном растворе по данным ЯМР спектроскопии разработана обобщенная статистико-термодинамическая модель гетероассоциации, учитывающая краевые эффекты при образовании гетерокомплексов. Проведенный анализ свидетельствует о том, что обобщенная модель в сравнении с базовой бесконечномерной моделью [10] более адекватно описывает процессы гетероассоциации ароматических красителей независимо от структурных особенностей хромофоров и термодинамических параметров самоассоциации молекул.

Выражаем благодарность Объединенному исследовательскому центру Лондонского университета за предоставленную возможность в Беркбек колледже использовать для измерений ЯМР-спектрометр «Bruker DRX» $(500 \mathrm{M} \Gamma \mathrm{L})$.

Работа частично финансировалась Международной программой INTAS (грант INTAS-9731753).
D. A. Veselkov, M. P. Eustigneev, D. B. Davies, V. V. Kodintsev, A. N. Veselkov

A generalized statistical-thermodynamical model of hetero-association of aromatic molecules in aqueous solution for the NMR data interpretation

Summary

On the ground of the basic hetero-association model derived previously, a generalized statistical-thermodynamical model of hetero-association of aromatic molecules has been developed for the NMR data interpretation. In the proposed model, unlike the basic one, the edge effects are taken into consideration, i.e. the dependence of the proton chemical shift on the position of the molecule situated inside, at the edge of the aggregate or in the hetero-stack. Both basic and generalized models were used for the analysis of hetero-association of acridine dye, proflavine $(P F)$, and phenanthridinium dye, ethidium bromide $(E B)$, in aqueous solution. The calculation of the association parameters of the molecules has been carried out using $H-N M R$ ( $500 \mathrm{MHz}$ ) experimental data. The experimental concentration and temperature dependence $s$ of the proton chemical shifts of interacting aromatic molecules have been studied. The parameters calculated according to the basic and generalized models are found to differ approximately by $30 \%$ and depend substantially on the magnitude of the equilibrium heteroassociation constant $K C$ - the larger the $K C$ value the higher the discrepancy between two models. The analysis of the structural and thermodynamical characteristics of the PF-EB complexation indicates the major role of dispersive interactions in stabilization of the PF-EB hetero-complex. KEY WORDS: statistical-thermodynamical model, proflavine, ethidium bromide, hetero-association, thermodynamics.

Д. О. Веселков, М. П. Євстигнєєв, Д. Б. Девіс, В. В. Кодинцев, о. Н. Веселков

Узагальнена статистико-термодинамічна модель гетероасоціації ароматичних молекул у водному розчині для аналізу даних ${ }^{1}$ H-ЯMPспектроскопії

\section{Резюме}

На основі базової моделі zетероасоціацї ароматичних молекул у водному розчині розроблено узагальнену статистико-термодинамінну модель дле інтерпретацї даних ЯМР-спектроскопії. В розzлянутій моделі, на відміну від базової, враховано крайові ефекти, тобто залежність хімінного зсуву протонів від полохення молекули в агрегаті - всередині, скраю або в zетеростику. Базова $і$ узагальнена моделі апробовано на прикладі zетероасоціації акридинового барвника профлавіну (PF) и фенантридинового барвника бромистого етидію (ЕB) у водно. му розчині. Разрахунок параметрів асочіацї молекул здійснено за данини Н-яМРспектроскопї (500 Мru). Досліджено експериментальні концентраційні і температурні залежності протонних хімічних зсувів взасмодіючих ароматичних молекул. встановлено, що разраховані параметри за базовою та узагальненою моделями відрізняються приблизно на $30 \%$ $i$ сутmево залежать від величини рівноважної константи гетероасоціацї $K_{\mathrm{C}}$ - при великих значеннях $K_{\mathrm{C}}$ похибка базової моделі зростас. Аналіз структурних $і$ термодинамічних характеристик комплексоутворення PF $і$ ЕB дозволяс зробити висновок иодо перевахного внеску дисперсійних взаємодій у стабілізацію гетерокомплексу $P F-E B$.

\section{СПИСОК ЛИТЕРАТУРЫ}

1. Adel A. L, Dorr R. T., Liddil J. D. The effect of anticancer 
drug sequence in experimental combination chemotherapy // Cancer Invest. -1993.-11.-P. 15-24.

2. Alberts D. S., Salmon S. E., Surwit E. A., Chen H. S. G., Moon $T$. E., Meyskens $F$. L. Combination chemotherapy (CRX) in vitro with the human-tumor stem-cell assay // Proc. Amer. Assoc. Cancer Res.-1 1981.-23.-P. 153-164.

3. Roberts J., Tumer N. Age and diet effects on drug action // Pharn. Ther.-1988.-37.-P. 111-149.

4. Jefferson $J . W$. Drug and diet interactions: avoiding therapeutic paralysis // J. Clin. Psych.-1998.-59.-P. 31-39.

5. Traganos $F$., Kapuscinsky J., Darzynkiewicz $Z$. Caffeine modulates the effects of DNA-intercalating drug in vitro: A flow cytometric and spectrophotometric analysis of caffeine interaction with novatrone, doxorubicin, ellipticine and doxorubicin analogue AD198 // Cancer Res.-1991._S1.P. 3682-3689.

6. Kapuscinsky J., Kimmel $M$. Thermodynamical model of mixed aggregation of intercalators with caffeine in aqueous solution // Biophys. Chem.-1993.-46.-P. 153-163.

7. Larsen R. W., Jasuja R., Hetzler R., Muraoka P. T., Andrada $V$. G., Jameson $D$. $M$. Spectroscopic and molecular modelling studies of caffeine complexes with DNA intercalators // Biophys. J. $-1996 .-70 .-$ P. $443-452$.

8. Davies D. B., Veselkov D. A., Diimant L. N., Veselkov A. N. Hetero-association of caffeine and aromatic drugs and their competitive binding with a DNA oligomer // Eur. Biophys. J.-2001.-30.-P. 354-356.

9. Davies D. B., Djimant L. N., Veselkov A. N. ${ }^{1} \mathrm{H}$ NMR investigation of self-association of aromatic drug molecules in aqueous solution // J. Chem. Soc. Faraday Trans.-1996.92.-P. 383-390.
10. Davies D. B., Veselkov D. A., Veselkov A. N. Structure and thermodynamics of the hetero-association of aromatic molecules in aqueous solution determined by NMR spectroscopy // Mol. Phys. 1999 . -97.-P. 439-451.

11. Bresloff J. L, Crothers D. M. Equilibrium studies of ethidiumpolynucleotide interactions // Biochemistry.-1981.-20.P. 3547-3553.

12. Albert A. The Acridines / Ed. L. E. Arnold.-New York, 1966. -604 p.

13. Итон Р. Дж., Барановский С. Ф., Осетров С. Г., Пахомов В. И., Болотин П. А., Дымант Л. Н., Дәвис Д. Б., Веселков А. Н. ${ }^{1}$ Н-ЯМР анализ комплексообразования антибиотика дауномицина с дезокситетрануклеотидом $5^{\prime}$ d(TpGpCpA) в водном растворе // Биополимеры и клетка. -1999.-15, № 2.-C. 87-96.

14. Delbarre A., Roques B. P., Le Pecq J. B., Lallemand J. Y., Xuong N. D. PMR studies of the self-association of DNA intercalating eflipticine derivatives in aqueous solution // Biophys. Chem.-1976.-4.-P. 275-279.

15. Chaires $J$. B. Thermodynamics of the daunomycin-DNA interaction: Ionic strength dependence of the enthalpy and entropy // Biopolymers.-1985.-24.-P. 403-419.

16. Ross $R$. D., Subramanian $S$. Thermodynamics of protein association reactions: forces contributing to stability // Biochemistry.-1981.-20.-P. 3096-3102.

17. Giessner-Prettre C., Pullman B. Quantum mechanical calculations of NMR chemical shifts in nucleic acids // Quart. Rev. Biophys. $-1987 .-20 .-$ P. $113-172$

УДК $541.49+539.1 .12 .43$ Надійшла до редакції 10.05 .2000 